\title{
How the Magnitude of Prey Genetic Variation Alters Predator-Prey Eco-Evolutionary Dynamics
}

\author{
Michael H. Cortez \\ Department of Mathematics and Statistics, Utah State University, Logan, Utah 84322 \\ Submitted August 31, 2015; Accepted April 14, 2016; Electronically published July 7, 2016 \\ Online enhancements: appendixes.
}

AвstraCt: Evolution can alter the stability and dynamics of ecological communities; for example, prey evolution can drive cyclic dynamics in predator-prey systems that are not possible in the absence of evolution. However, it is unclear how the magnitude of additive genetic variation in the evolving species mediates those effects. In this study, I explore how the magnitude of prey additive genetic variation determines what effects prey evolution has on the dynamics and stability of predator-prey systems. I use linear stability analysis to decompose the stability of a general eco-evolutionary predator-prey model into components representing the stabilities of the ecological and evolutionary subsystems as well as the interactions between those subsystems. My results show that with low genetic variation, the cyclic dynamics and stability of the system are determined by the ecological subsystem. With increased genetic variation, disruptive selection always destabilizes stable communities, stabilizing selection can stabilize or destabilize communities, and prey evolution can alter predator-prey phase lags. Stability changes occur approximately when the magnitude of genetic variation balances the (in)stabilities of the ecological and evolutionary subsystems. I discuss the connections between my stability results and prior results from the theory of adaptive dynamics.

Keywords: community dynamics, population dynamics, heritability, adaptive dynamics.

\section{Introduction}

An important area of research at the intersection of ecology and evolution is how genotypic diversity and evolution alter the population- and community-level dynamics of ecological systems. Genotypic diversity and evolution can influence the stability and structure of ecological communities, species coexistence, and ecosystem-level processes (Abrams 2000; Whitham et al. 2003; Hughes et al. 2008; Bolnick et al. 2011). Predator-prey systems are one class of systems where the interplay between ecological and evolutionary processes has been observed to alter the population-level

\footnotetext{
* E-mail: michael.cortez@usu.edu.
}

Am. Nat. 2016. Vol. 188, pp. 329-341. (C) 2016 by The University of Chicago. 0003-0147/2016/18803-56497\$15.00. All rights reserved. DOI: $10.1086 / 687393$ dynamics and, in some cases, drive dynamics that cannot occur in the absence of evolution.

In the absence of evolution, predator-prey systems are predicted to exhibit cycles where peaks in prey abundance precede peaks in predator abundance by less than a quarter of the cycle period (Bulmer 1975). In contrast, in experimental systems prey evolution has been observed to drive antiphase cycles, where prey peaks precede predator peaks by a half period (Yoshida et al. 2003; Becks et al. 2010), or cryptic cycles, where predator abundance fluctuates and prey abundance remains effectively constant (Yoshida et al. 2007). Hence, prey evolution can drive ecological dynamics that differ qualitatively from those exhibited in the absence of evolution. This experimental work is complimented by theory that identifies the biological conditions under which antiphase and cryptic cycles arise (Jones and Ellner 2007; Yoshida et al. 2007; Cortez and Ellner 2010); prey evolution is predicted to drive antiphase cycles when increased prey defense comes at the cost of a large decrease in prey growth rate, and cryptic cycles are predicted to occur when the cost is small. Increased genetic variation in prey populations has also been observed to alter the stability of experimental predator-prey systems. For example, increased algal genetic variation in one rotifer-algal system (Becks et al. 2010) caused an initially stable system to undergo cyclic oscillations (fig. 1). In another rotifer-algal system (Steiner and Masse 2013), increased algal genetic variation was stabilizing and decreased the amplitude of the prey oscillations.

While this empirical and theoretical work shows that prey evolution can have a large impact on the population dynamics and stability of predator-prey systems, evolution does not always alter system behavior. For example, in the Becks et al. (2010) algal-rotifer system mentioned above, seeding the prey population with low amounts of genetic variation does not alter the stability of the system, and the populations converge to a steady state (fig. $1 A, 1 B$ ). In contrast, seeding the prey population with large amounts of genetic variation destabilizes the system and yields sustained antiphase oscillations (fig. 1C, 1D). Hence, while evolution occurs in both cases, qualitative changes in system behavior 

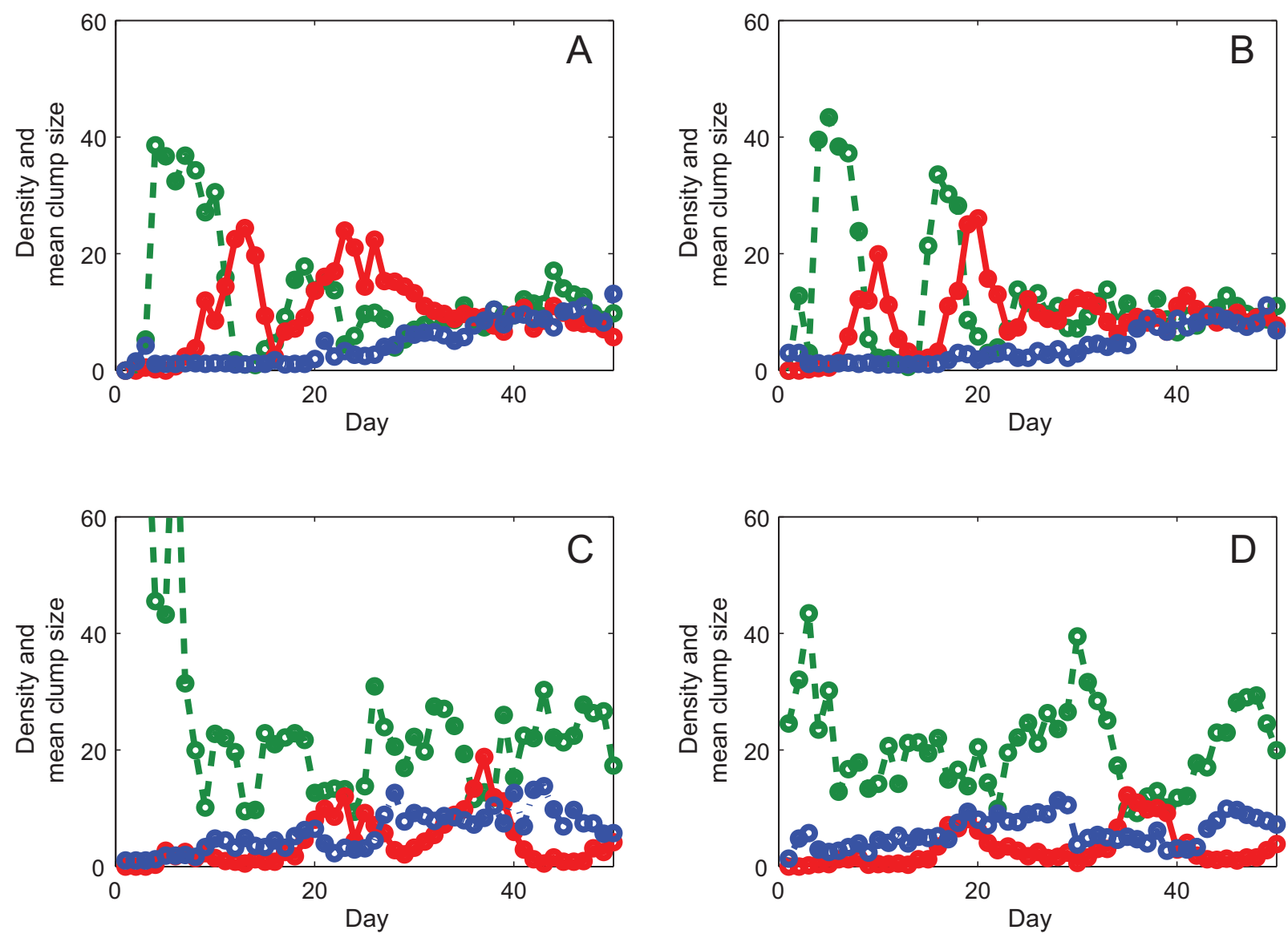

Figure 1: Increased prey additive genetic variation destabilizes an experimental algal-rotifer system. Data are from the Becks et al. (2010) study. All panels show rotifer density (Brachionus calyciflorus, rotifers $\mathrm{mL}^{-1}$; solid red), algal density (Chlamydomonas reinhardtii, $10^{4}$ cells $\mathrm{mL}^{-1}$; dashed green), and mean algal clump size (cells per palmelloid colony; dashed-dotted blue). The system converges to a steady state when seeded with low prey genetic variation $(A, B)$ and exhibits sustained oscillations when seeded with high amounts of genetic variation $(C, D)$.

occur only when the amount of genetic variation in the prey population is sufficiently large. These empirical results motivate the question, How much genetic variation is needed for evolution to alter the stability of a predator-prey system? Or, more generally, How does the magnitude of genetic variation influence the effects evolution has on population-level dynamics?

To answer these questions, we need theory about how increasing the standing genetic variation in a population from very small to very large amounts influences the effects evolution has on the ecological dynamics of a system. The current bodies of eco-evolutionary theory can be thought of as lying on a continuum where the amount of standing genetic variation in a population ranges between being very small to very large. At the extreme where standing genetic variation is very low, the theory of adaptive dynamics (Marrow et al. 1996; Geritz et al. 1998) and other work on mutation- limited evolution (Doebeli 1997) focus on the limit where ecological changes (e.g., changes in population sizes) occur much faster than evolutionary changes. At the other extreme where standing genetic variation is very high, theory based on quantitative genetic models focuses on the limit where evolutionary changes occur faster than ecological changes (Cortez and Ellner 2010). In between these two extremes is a body of theory based on quantitative genetic models with moderate amounts of standing genetic variation (Abrams et al. 1993; Abrams and Matsuda 1997) and clonal models where prey evolution occurs as clonal frequencies fluctuate in time (Jones and Ellner 2007; Jones et al. 2009; Becks et al. 2010). While these bodies of theory span the full range of amounts of standing genetic variation, it is not clear how to connect the different results and predictions from each body of theory. Hence, there is need for theory that explains how the magnitude of genetic variation 
in a population mediates how evolution shapes the populationand community-level dynamics of eco-evolutionary systems (Bolnick et al. 2011).

To begin building such a theory, I explore how the dynamics and stability of a general eco-evolutionary predatorprey model with an evolving prey defense depend on the magnitude of additive prey genetic variation. With the model I answer three questions: (1) Under what conditions does increased additive genetic variation cause prey evolution to alter system stability? (2) How much additive genetic variation is needed to alter system stability? and (3) How does the magnitude of prey additive genetic variation influence the phase lags of predator-prey cycles? I use linear stability analysis (i.e., the Jacobian) to decompose the stability of equilibria in the full eco-evolutionary system into components that define the stability of the ecological subsystem, the stability of the evolutionary subsystem, and interactions between those subsystems. Abrams and Matsuda (1997) used a similar approach to study when prey evolution drove cyclic dynamics in a specific predator-prey model. Because my work focuses on a predator-prey model with general functional forms, my work extends the results in Abrams and Matsuda (1997) and yields predictions that apply across systems about how the stabilities of the ecological and evolutionary subsystems contribute to the stability of the whole system and how those effects are mediated by the amount of standing prey genetic variation. In addition, this work identifies connections between stability results derived with the theory of adaptive dynamics and those derived via quantitative genetic approaches.

\section{Predator-Prey Model with Prey Evolution}

This study builds on the analysis of the eco-evolutionary predator-prey model in Cortez and Ellner (2010). That study addressed only how frequency-independent selection alters ecological dynamics in the limit where prey genetic variation is very large (i.e., fast evolutionary dynamics). Cortez and Weitz (2014) and Cortez (2015) extended that model to include predator-prey coevolution, but those studies also focused only on the limit where the genetic variation in both species is very large. This study differs from that previous work in that it considers frequency-dependent selection and identifies how prey evolution alters the ecological dynamics of the system for all possible amounts of genetic variation.

The model describes the changes in the total prey $(x)$ and predator $(y)$ densities and the mean prey trait $(\alpha)$. There is a trade-off between susceptibility to predation (defense) and prey growth rate. Smaller trait values imply reduced susceptibility to predation, which comes at the cost of reduced growth rate; for example, reduced susceptibility to consumption by rotifers results in reduced competitive ability to obtain resources (Yoshida et al. 2003, 2007; Kasada et al. 2014). The model is

$$
\begin{aligned}
\frac{\mathrm{d} x}{\mathrm{~d} t} & =F\left(x, \alpha_{i}, \alpha\right)-\left.G\left(x, y, \alpha_{i}, \alpha\right)\right|_{\alpha_{i}=\alpha}, \\
\frac{\mathrm{d} y}{\mathrm{~d} t} & =H(x, y, \alpha)-D(y), \\
\frac{\mathrm{d} \alpha}{\mathrm{d} t} & =\left.V \frac{\partial}{\partial \alpha_{i}}\left(\frac{1}{x} \frac{\mathrm{d} x}{\mathrm{~d} t}\right)\right|_{\alpha_{i}=\alpha},
\end{aligned}
$$

where $F$ is the growth rate of the prey in the absence of predation, $G$ is the predation rate, $H$ is the composition of the predation rate and the predator to prey conversion, and $D$ is the death rate of the predator. Note that the functions are often written in their per capita forms, for example, $F=$ $x f\left(x, \alpha_{i}, \alpha\right)$ and $G=x g\left(x, y, \alpha_{i}, \alpha\right)$. The variable $\alpha_{i}$ represents the trait value of an individual prey. Because the growth rate of the total prey population is determined by the mean trait value, the first line of model (1) is evaluated at $\alpha_{i}=\alpha$. I assume that $F$ and $G$ are increasing functions of $\alpha_{i}$ and $\alpha$; that $G$ and $H$ are increasing functions of $x, y$, and $\alpha$; and that $D$ is an increasing function of $y$. These assumptions define the trade-off between prey growth and susceptibility to predation, which in general is density and frequency dependent. In the following, subscripts denote partial derivatives, that is, $F_{x}=\partial F / \partial x$.

The evolutionary dynamics of the model $(\mathrm{d} \alpha / \mathrm{d} t$ equation) follow from the quantitative genetics approach derived in Lande $(1976,1982)$ and Abrams et al. (1993). In that equation, the rate of change of the mean trait value is proportional to the additive genetic variation in the prey population, $V$, and the individual fitness gradient,

$$
\left.\frac{\partial}{\partial \alpha_{i}}\left(\frac{1}{x} \frac{\mathrm{d} x}{\mathrm{~d} t}\right)\right|_{\alpha_{i}=\alpha} .
$$

The fitness gradient depends on the individual's phenotype, $\alpha_{i}$, because selection is frequency dependent.

The additive prey genetic variation determines the speed of the evolutionary dynamics in model (1). When $V$ is very small, the evolutionary dynamics of model (1) are much slower than the ecological dynamics. In this case, standing genetic variation is mutation limited. When $V$ is close to 1 $(V \approx 1)$, the speeds of the ecological and evolutionary dynamics of model (1) are comparable. This can occur either when selection is weak relative to mutation or when all possible genotypes are present (possibly at low density), and evolution occurs as genotype frequencies change (e.g., clonal systems). Finally, when $V$ is very large $(V \gg 1)$, the evolutionary dynamics of model (1) are much faster than the ecological dynamics. While less likely to occur in natural systems, this limit has been studied previously to gain insight 
about the dynamics that occur when $V \approx 1$ (Cortez and Ellner 2010).

\section{Model Generality and Assumptions}

The derivations in Lande $(1976,1982)$ and Abrams et al. (1993) assume that the trait is normally distributed with a fixed additive genetic variation. In addition, gradient dynamic models, like model (1), do not explicitly model the relationship between genotype and phenotype, implying that they may yield an incomplete picture when specifics about genetic processes matter. Despite these underlying assumptions, gradient dynamic models are a good starting point for studying eco-evolutionary dynamics because they are analytically tractable and approximate the dynamics of many kinds of evolutionary models that do not satisfy the assumptions (Abrams 2001, 2005). In addition, the model is exact or yields a good quantitative and qualitative approximation to many different kinds of systems with large additive genetic variation. This includes systems with continuous trait values where individual fitness is a linear or quadratic function of the individual's trait value (Abrams et al. 1993) and clonal systems with discrete trait values (Cortez and Weitz 2014). The models are also reasonable approximations for systems with disruptive selection, provided there is random mating and additive contributions from many loci (Turelli and Barton 1994).

Model (1) assumes that the additive prey genetic variation is constant, but in general genetic variation can change over time. Because my approach focuses on the stability of equilibrium points, all of my analytical results apply to models where the genetic variation changes over time, provided the value of $V$ in model (1) is set equal to the equilibrium value of the genetic variation in the model with variable genetic variation; for details, see appendix A, section A1 (apps. A-E are available online). Hence, changes in the magnitude of genetic variation can cause differences in model behavior far from the equilibrium, but those changes will not affect the local stability results presented in this study. Note that in simulations I use $V(\alpha)=\left(\alpha-\alpha_{\min }\right)\left(\alpha_{\max }-\alpha\right)$, where $\alpha_{\min }$ and $\alpha_{\max }$ are the minimum and maximum allowable mean trait values, respectively. This form is appropriate, for example, for clonal populations where the prey have discrete phenotypes (Cortez and Weitz 2014). In this case, the additive genetic variation converges to 0 as the mean trait approaches either extreme; if the mean equals an extreme value, then all individuals have that extreme value, and there is no genetic variation.

Finally, because additive genetic variation is the product of phenotypic variation and narrow-sense heritability, changes in $V$ can be interpreted as changes in either or both quantities. The functions in model (1) do not depend on phenotypic variation; hence, changes in phenotypic variation or narrowsense heritability have the same effect. In systems where the functional forms do depend on phenotypic variation (e.g., Schreiber et al. 2011; Patel and Schreiber 2015), changes in phenotypic variation and narrow-sense heritability may have quantitatively different effects. Nonetheless, all of my results remain unchanged for those systems provided that changes in $V$ represent changes in heritability. Furthermore, if changes in $V$ represent changes in phenotypic variation, all of my analytical results remain unchanged, provided the Jacobian entries do not change sign; for details, see appendix A, section A1.

\section{Model Analysis via Linearization}

To explore how the magnitude of prey genetic variation influences system stability, I study the stability of equilibria of model (1) via the Jacobian, J(fig. 2). The Jacobian determines whether small changes in the population densities or mean trait value will grow (implying instability of an equilibrium point) or decrease (implying equilibrium stability). Interpretations and signs for the entries of $J$ are given in table 1. Additional details about the interpretation of $J$ and its entries are given in appendix A, section A2. Throughout I assume that $J$ has a negative determinant. When the determinant of $J$ is positive, the equilibrium point is always un-

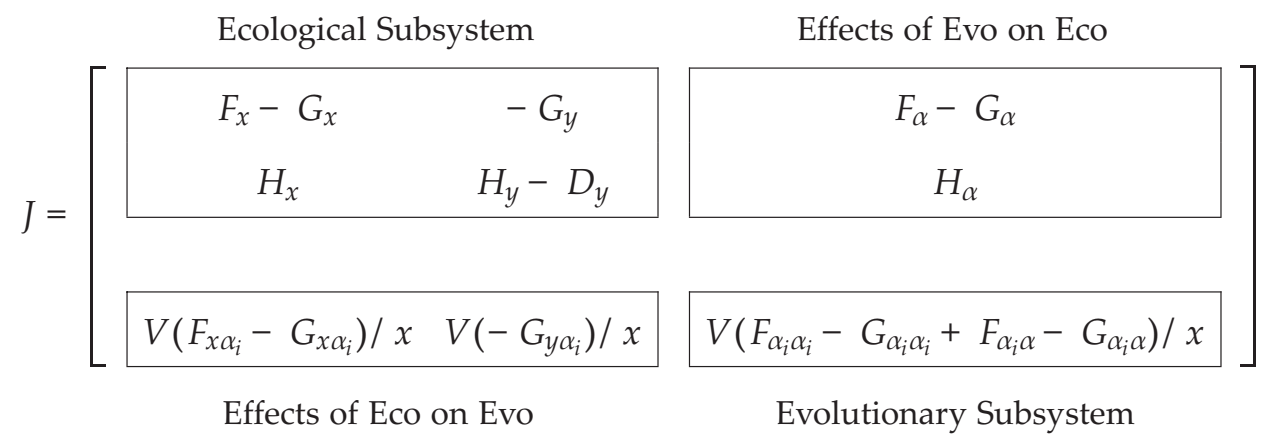

Figure 2: The Jacobian of model (1) evaluated at eco-evolutionary coexistence equilibrium point. 
Table 1: Interpretation and signs of Jacobian entries

\begin{tabular}{llc}
\hline Value & \multicolumn{1}{c}{ Description of effect } & Sign $^{\text {a }}$ \\
\hline$F_{x}-G_{x}$ & Effect of prey density on prey growth rate & \pm \\
$-G_{y}$ & Increased predation decreases prey growth rate & - \\
$H_{x}$ & Increased prey consumption increases predator growth rate & + \\
$H_{y}-D_{y}$ & Intraspecific competition decreases predator growth rate & - \\
$\xi=\left(F_{\alpha_{i} \alpha_{i}}-G_{\alpha_{i} \alpha_{i}}+F_{\alpha_{i} \alpha}-G_{\alpha_{i} \alpha}\right) / x$ & Stable $(\xi<0)$ or unstable $(\xi>0)$ evolutionary subsystem; & \pm \\
$F_{\alpha}-G_{\alpha}$ & stabilizing $(\xi<0)$ or disruptive $(\xi>0)$ selection & \pm \\
$H_{\alpha}$ & Effect of mean prey defense on individual fitness & + \\
$F_{x \alpha_{i}}-G_{x \alpha_{i}}$ & Decreased defense increases predator growth rate & + \\
$-G_{y \alpha_{i}}$ & Increased prey density decreases selection for defense & + \\
\hline
\end{tabular}

a Values that can have either sign are denoted by \pm .

stable and not biologically meaningful; for details, see appendix A, section A2.

Importantly, the Jacobian decomposes into biologically interpretable components. The top left box determines the stability of the ecological subsystem in the absence of evolution. If the ecological subsystem is unstable, predatorprey cycles occur in the absence of evolution. If the ecological subsystem is stable, stable coexistence occurs in the absence of evolution.

The bottom right box of the Jacobian (hereafter denoted $\left.\xi=\left[F_{\alpha_{i} \alpha_{i}}-G_{\alpha_{i} \alpha_{i}}+F_{\alpha_{i} \alpha}-G_{\alpha_{i} \alpha}\right] / x\right)$ determines the concavity of the trade-off between prey growth and defense (Cortez and Ellner 2010). When $\xi<0$, the trade-off is concave down, implying decelerating costs for defense. When $\xi>0$, the trade-off is concave up, implying accelerating costs for defense. The quantity $\xi$ also determines the stability of the evolutionary subsystem when the population densities are fixed. Let $\alpha^{*}$ be the equilibrium trait value for some fixed population densities. If $\xi<0$, then $\alpha^{*}$ is an attractor and selection drives the mean trait value toward $\alpha^{*}$. In this case, the evolutionary subsystem is stable. I refer to this case as stabilizing selection. If $\xi>0$, then $\alpha^{*}$ is a repeller and selection drives the mean trait value away from $\alpha^{*}$ and toward one of the extreme trait values (either $\alpha_{\min }$ or $\alpha_{\max }$ ). Which extreme the trait is driven toward depends on the initial mean trait value: if the initial mean trait value is smaller (larger) than $\alpha^{*}$, then selection will drive the mean trait value to $\alpha_{\min }\left(\alpha_{\max }\right)$. In this case, the evolutionary subsystem is unstable. I refer to this case as disruptive selection.

The off-diagonal boxes in the Jacobian determine the effects evolution has on ecological processes (top right box) and the effects ecological processes have on evolution (bottom left box). Three entries have assumed fixed signs: $H_{\alpha}>0$ because decreased defense increases the predation rate, $F_{x \alpha_{i}}-G_{x \alpha_{i}}>0$ because increased prey density results in increased selection for higher prey growth rates, and $F_{x \alpha_{i}}-$ $G_{x \alpha_{i}}<0$ because increased predator density results in selection for higher defense. The fourth term, $F_{\alpha}-G_{\alpha}$, determines whether individual prey fitness increases or decreases with higher mean defense. Increased individual fitness with higher mean defense $\left(F_{\alpha}-G_{\alpha}<0\right)$ occurs when prey have a shared defense (e.g., an excreted chemical defense) or when more defended prey competitively interfere less with conspecifics. Decreased individual fitness with higher mean defense $\left(F_{\alpha}-G_{\alpha}>0\right)$ occurs when less defended prey produce a shared resource.

Using the Jacobian, I determine how increasing the magnitude of additive genetic variation $(V)$ changes the dynamics and stability of model (1). Stability changes occur when increasing $V$ causes either an initially stable system to cycle (destabilization) or an initially cyclic system to converge to an equilibrium (stabilization). In the following, I focus on the following cases: the ecological subsystem is stable and the evolutionary subsystem is unstable, the ecological subsystem is unstable and the evolutionary subsystem is stable, and the ecological and evolutionary subsystems are both stable. This categorization identifies how the stabilities of each subsystem influence the stability of the whole system and how those effects are mediated by the magnitude of prey additive genetic variation.

\section{Results}

\section{Dependence of System Stability on Genetic Variation}

I first explore how prey additive genetic variation affects system stability. This work identifies how much additive genetic variation is needed for prey evolution to destabilize or stabilize a system and under what conditions destabilization and stabilization occur. Additional details about results are presented in the sections of appendixes A and B referenced below.

First, for low genetic variation ( $V$ is small), the stability of the system is always the same as the ecological subsystem; see appendix A, section A3. This means that regardless of the stabilities of the ecological and evolutionary 
subsystems and their interactions, the stability of the ecological subsystem dominates at low genetic variation. Hence, prey evolution can alter system stability only with sufficiently high amounts of genetic variation.

Consider systems where the ecological subsystem is stable and the evolutionary subsystem is unstable $(\xi>0$, disruptive selection); see appendix $B$, section $B 1$. The system is stable for low genetic variation (left side of fig. $3 A$ ), meaning that the ecological subsystem stabilizes the unstable evolutionary dynamics at low genetic variation. For sufficiently high genetic variation, disruptive selection destabilizes the system and causes population cycles (right side of fig. 3 ). Hence, the instability of the evolutionary subsystem overpowers the stability of the ecological subsystem provided the amount of genetic variation is sufficiently high.

Now consider systems where the ecological subsystem is unstable and the evolutionary subsystem is stable $(\xi<0$, stabilizing selection); see appendix B, section B2. Population cycles occur when genetic variation is low because the ecological subsystem is unstable (left side of fig. $4 A, 4 B$ ). At higher levels of genetic variation, prey evolution can stabilize the system and cause the cycles to cease. Stabilization occurs when individual fitness increases with higher mean defense (e.g., shared chemical defenses; $F_{\alpha}-G_{\alpha}<0$ ) and the trade-off between prey defense and growth is nearly linear ( $\xi$ is small). One way to think about why stabilization occurs is that the shared defenses dampen the ecological and evolutionary responses of the prey to changes in predator density, which dampens the predator fluctuations; sufficient dampening stabilizes the system. Stabilization occurs either for (1) all $V$ greater than some minimum value (fig. $4 A$ )

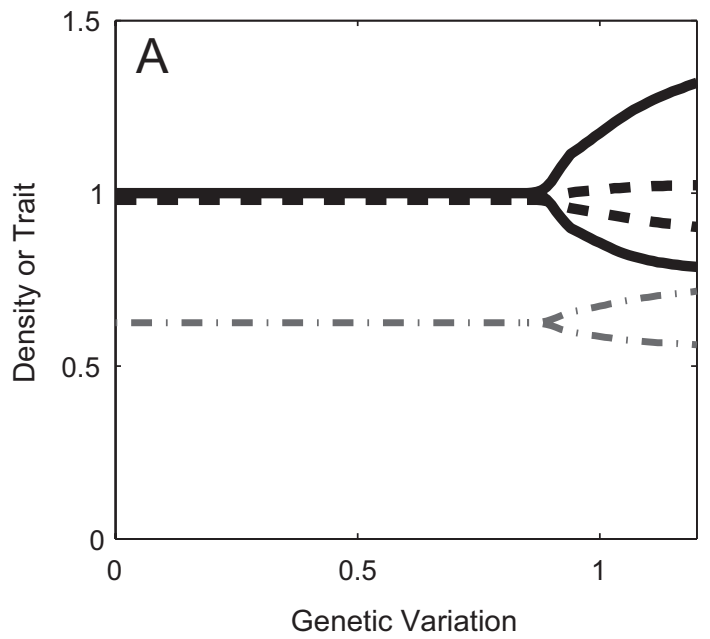

or (2) a finite range of values (fig. $4 B$ ). The former occurs when $F_{\alpha}-G_{\alpha}$ is large and negative (e.g., effective, shared chemical defense), and the latter occurs when $F_{\alpha}-G_{\alpha}$ is small and negative (e.g., weakly effective, shared chemical defense).

Last, consider systems where the ecological and evolutionary subsystems are stable; see appendix B, section B3. Because each subsystem is stable, the expectation is that the full eco-evolutionary system is stable. This is always the case for low genetic variation (left side of fig. $4 D, 4 E$ ). However, at higher levels of genetic variation, prey evolution can destabilize the system and cause population cycles. Destabilization occurs when individual fitness increases with lower mean defense (low-defense prey share resources; $\left.F_{\alpha}-G_{\alpha}>0\right)$ and the trade-off between prey defense and growth is nearly linear ( $\xi$ small). One way to think about why destabilization occurs is that shared resources allow prey with greater defense to increase in abundance. This can cause fluctuations in predator density that lead to sustained cycles. Destabilization occurs either for (1) all $V$ greater than some minimum value (fig. 4D) or (2) a finite range of values (fig. $4 E$ ). The former occurs when $F_{\alpha}-G_{\alpha}$ is large and positive (e.g., low-defense prey share many resources), and the latter occurs when $F_{\alpha}-G_{\alpha}$ is small and positive (e.g., lowdefense prey share few resources).

In summary, the stability of the ecological subsystem dominates when genetic variation is low. Under disruptive selection, increased genetic variation always destabilizes a stable ecological system. Under stabilizing selection, increased genetic variation can stabilize or destabilize a system; shared defenses are stabilizing, whereas defenses that come at the

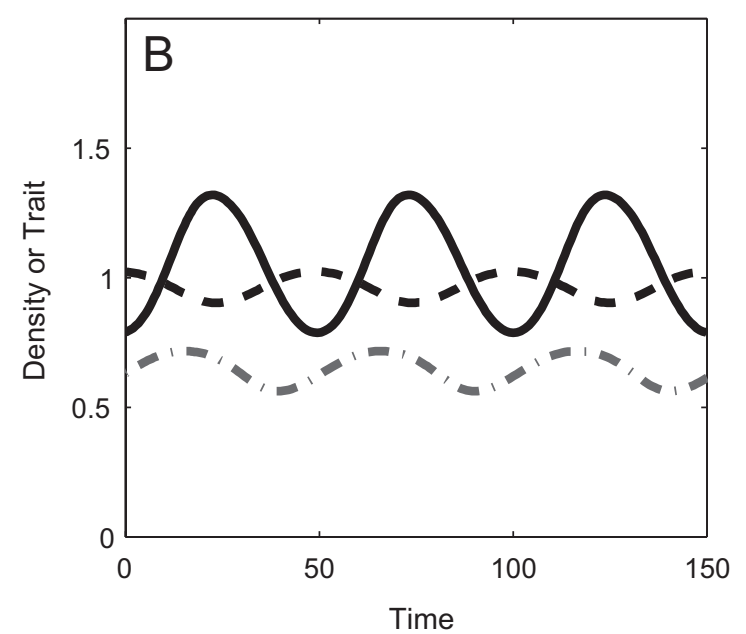

Figure 3: With sufficiently large amounts of additive genetic variation, disruptive selection always destabilizes a stable ecological system. $A$, Maximum and minimum long-term predator density (solid black), prey density (dashed black), and mean prey trait (dashed-dotted gray). For each genetic variation value, a single curve for each variable denotes the stable equilibrium value, whereas two curves denote the maximum and minimum values during the cycle. $B$, Numerical example of the cycle at $V=1.2$; the predator-prey phase lag is approximately one-half of the cycle period. See appendix E for model and parameter values. 

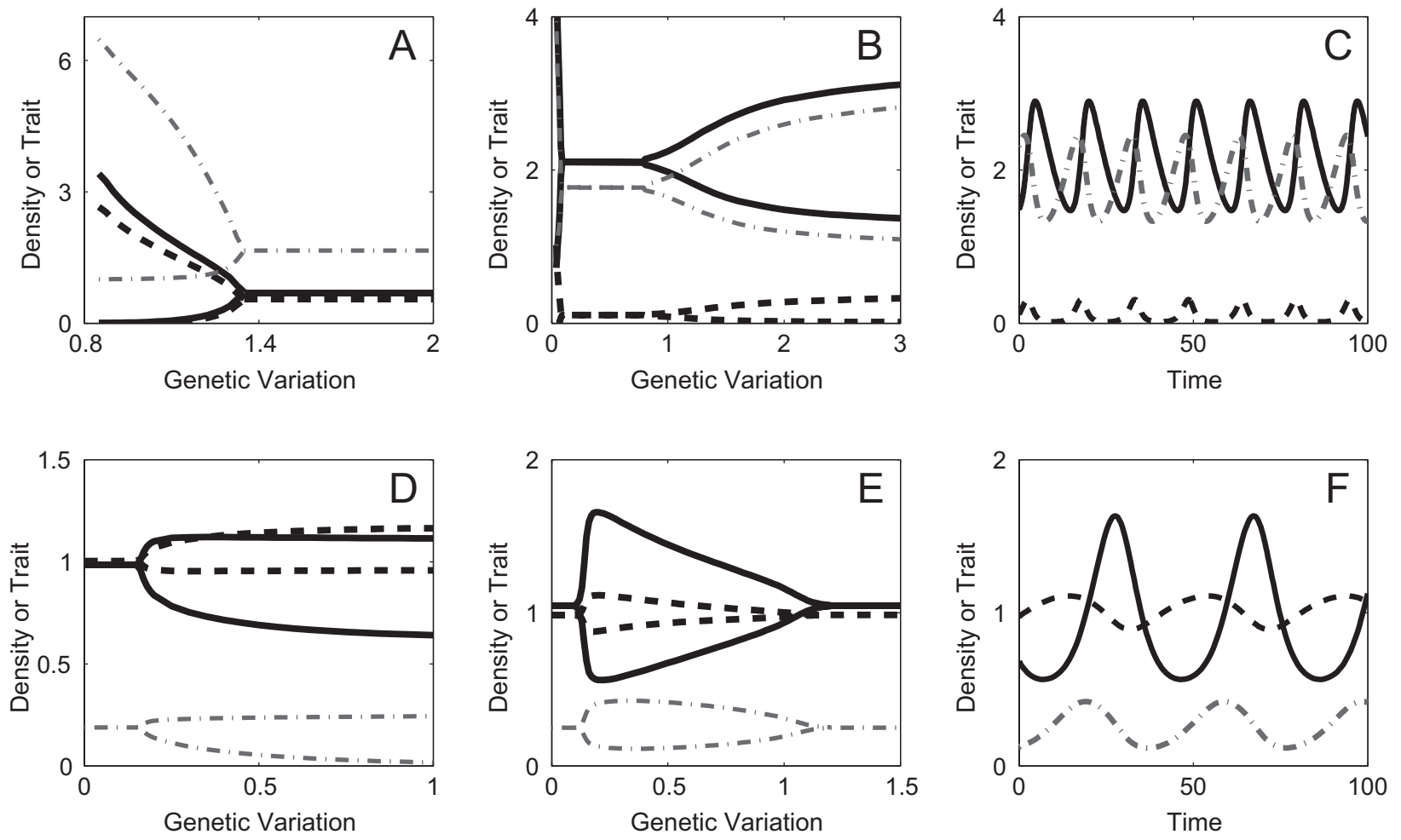

Figure 4: With increased additive genetic variation, stabilizing selection can stabilize ecological oscillations $(A-C)$ or destabilize a stable ecological system $(D-F)$. Destabilization occurs when individual fitness decreases with higher mean defense $\left(F_{\alpha}-G_{\alpha}>0\right)$, and stabilization occurs when individual fitness decreases with higher mean defense $\left(F_{\alpha}-G_{\alpha}<0\right)$. (De)stabilization occurs for all sufficiently large genetic variation when $F_{\alpha}-G_{\alpha}$ is large in magnitude $(A, D)$ and for intermediate genetic variation values when $F_{\alpha}-G_{\alpha}$ is small in magnitude $(B, E)$. $A, B, D, E$, Maximum and minimum long-term predator density (solid black), prey density (dashed black), and mean prey trait (dashed-dotted gray). For each genetic variation value, a single curve for each variable denotes the stable equilibrium value, and two curves denote the maximum and minimum values during the cycle. $C$, Numerical example of the cycle at $V=0.05$ in $A$; the predator-prey phase lag is less than a quarter period. $F$, Numerical example of the cycle at $V=0.25$ in $D$; the predator-prey phase lag is between one-quarter and one-half of a period. See appendix E for models and parameter values.

cost of reduced sharing of resources among conspecifics are destabilizing.

\section{(De)stabilization Occurs When the Amount of Genetic Variation Balances Subsystem (In)stabilities}

I now identify how much genetic variation is needed for disruptive selection to destabilize a system (fig. $3 A$ ) or for stabilizing selection to stabilize a system (fig. $4 A, 4 B$ ). The critical value at which (de)stabilization occurs is presented in equation (B6) of appendix B, section B5, but that equation is difficult to interpret biologically. To gain some insight, I derive an approximation for the critical value in the limit where the ecological subsystem is strongly stable or unstable. I discuss the main conclusions from that work here; for details, see appendix B, section B5.

The critical magnitude of genetic variation is approximately $V=-S_{\text {eco }} / \xi$. Here, $\xi$ is the stability or instability of the evolutionary subsystem, and $S_{\text {eco }}$ is the stability or in- stability of the ecological subsystem (measured by the leading eigenvalue of the Jacobian of the ecological subsystem; for stable subsystems this is the subsystem resilience sensu Pimm and Lawton [1977]). Rewriting this equation as $V \xi=-S_{\text {eco }}$ reveals that at the critical value of $V$, the (in)stabilities of the ecological and evolutionary subsystems are balanced. Hence, destabilization occurs when the stability of the ecological subsystem is counteracted by an equally strong destabilizing evolutionary force. Stabilization occurs when the instability of the ecological subsystem is counteracted by an equally strong stabilizing evolutionary force.

This result means that changes in system stability at low genetic variation imply that the ecological subsystem is weakly stable or weakly unstable. Moreover, evolution alters system stability over a wider range of magnitudes of genetic variation when the ecological subsystem is weakly (un)stable. Changes in system stability at high genetic variation imply that the ecological subsystem is strongly stable or 
strongly unstable. Moreover, when the ecological subsystem is strongly (un)stable, large magnitudes of genetic variation are needed for evolution to alter system stability.

\section{Effects of Genetic Variation on Predator-Prey Phase Lags}

In the absence of evolution, prey peaks precede predator peaks by less than a quarter of the cycle period (Bulmer 1975). Prey evolution can drive cycles with lags greater than a quarter period; such lags are considered populationlevel signatures of prey evolution (Yoshida et al. 2003; Jones and Ellner 2007; Cortez and Ellner 2010). Here, I explore how the amount of prey genetic variation affects predatorprey phase lags. To do this, I compute the lags at the values of $V$ where changes in stability occur, that is, where there is a transition from a stable equilibrium to cyclic dynamics. Mathematically, these points are known as Hopf bifurcations. I focus on lags at Hopf bifurcations because the lags at Hopf points can be computed analytically and, in numerical simulations, the lags at Hopf points approximate the lags at other values of $V$. Details about the following are given in appendix C.

If stabilization or destabilization occurs at low genetic variation ( $V$ small), then the phase lag is always a quarter period or less; for details, see appendix $\mathrm{C}$, section $\mathrm{C} 1$. For example, the cycle in figure $4 C$ that is born at the smaller critical value in figure $4 B(V \approx 0.083)$ has a phase lag approximately equal to one-quarter of the period. This result implies that prey evolution does not alter predator-prey phase lags in systems with weakly stable or weakly unstable ecological subsystems, even though evolution influences the stability of those systems over a large range of magnitudes of genetic variation.

When stabilization or destabilization occurs at larger magnitudes of genetic variation, the phase lags can be greater than a quarter period. There are two cases: (1) the lags are between one-quarter and one-half of the period (fig. 5C) and (2) the lags are greater than one-half of the period (fig. $5 F$ ). Mathematically, phase lags between one-quarter and one-half of the period arise when (i) $\left(F_{x \alpha_{i}}-G_{x \alpha_{i}}\right)\left(H_{\alpha}\right)>0$ is large or (ii) $H_{y}-D_{y}-H_{\alpha}\left(F_{x_{i}}-G_{x \alpha_{i}}\right) / \xi>0$; see appendix $\mathrm{C}$, sections C3.1 and C2, respectively. Condition (ii) was reported in Cortez and Ellner (2010), but because condition (i) is more biologically likely I focus on it and refer the reader to appendix C, section C2, for details about condition (ii). Biologically, condition (i) is satisfied when prey defense is highly effective against predation ( $H_{\alpha}$ is large), that is, when defended prey are nearly invulnerable to predation. Examples of cycles with lags between one-quarter and onehalf of the period driven by an effective defense are shown in figure $4 F$ for stabilizing selection and in figures $3 B, 5 A$, and $5 B$ for disruptive selection.
In very rare cases, the phase lags can be greater than half of the period; for details, see appendix C, section C3.2. In these rare cases the peaks in the predator density precede the peaks in prey density (fig. $5 D$ ), and the cycle has a clockwise orientation in the predator-prey phase plane (fig. $5 E$ ). Such cycles arise only when the negative direct effect of increased prey density on the selective pressure for defense is smaller in magnitude than the positive indirect effect. Specifically, increased prey density has a negative direct effect on the selective pressure for defense $\left(F_{x \alpha_{i}}-G_{x \alpha_{i}}>0\right)$. Increased prey density also has a positive indirect effect because increases in prey density cause increases in predator density, which cause increases in the selective pressure for defense. When the indirect effect is larger in magnitude than the direct effect, phase lags greater than a half period are possible. However, in numerical simulations, cycles with lags greater than a half period occur only in very small regions of parameter space. This suggests that such cycles are unlikely to be observed in natural systems. In addition, in all simulations the predator cycles are much smaller in amplitude than the prey cycles (fig. 5D). These are cryptic cycles (Yoshida et al. 2007), but because the prey oscillations have the larger amplitude, the cycles in figure $5 D$ are the reverse of those observed in experimental systems (Bohannan and Lenski 1999; Yoshida et al. 2007).

In summary, cycles arising at low genetic variation have phase lags less than a quarter period. Cycles arising with higher genetic variation can have lags greater than a quarter period. Lags between one-quarter and one-half of the period (fig. 5C) occur when prey defense is highly effective. Lags greater than a half period (fig. $5 F$ ) are also possible, but they are unlikely to be observed in empirical systems. These results suggest that in addition to being signatures of prey evolution (Yoshida et al. 2003; Jones and Ellner 2007; Cortez and Ellner 2010), lags greater than a quarter period are signatures of high prey genetic variation.

\section{Discussion}

Previous experimental (Yoshida et al. 2003, 2007; Becks et al. 2010; Steiner and Masse 2013) and theoretical (Abrams and Matsuda 1997; Jones et al. 2009; Cortez and Ellner 2010) studies have shown that prey evolution can alter the stability and dynamics of predator-prey systems. This study builds on that work by identifying how those effects depend on or are mediated by the amount of standing additive genetic variation in the prey population. At a basic level, my results show that evolution has very little effect when genetic variation is lacking and that evolution can have large effects when genetic variation is sufficiently high, that is, increased genetic variation enables evolution to alter population-level dynamics. However, my results also identify how these effects depend on the processes occurring 

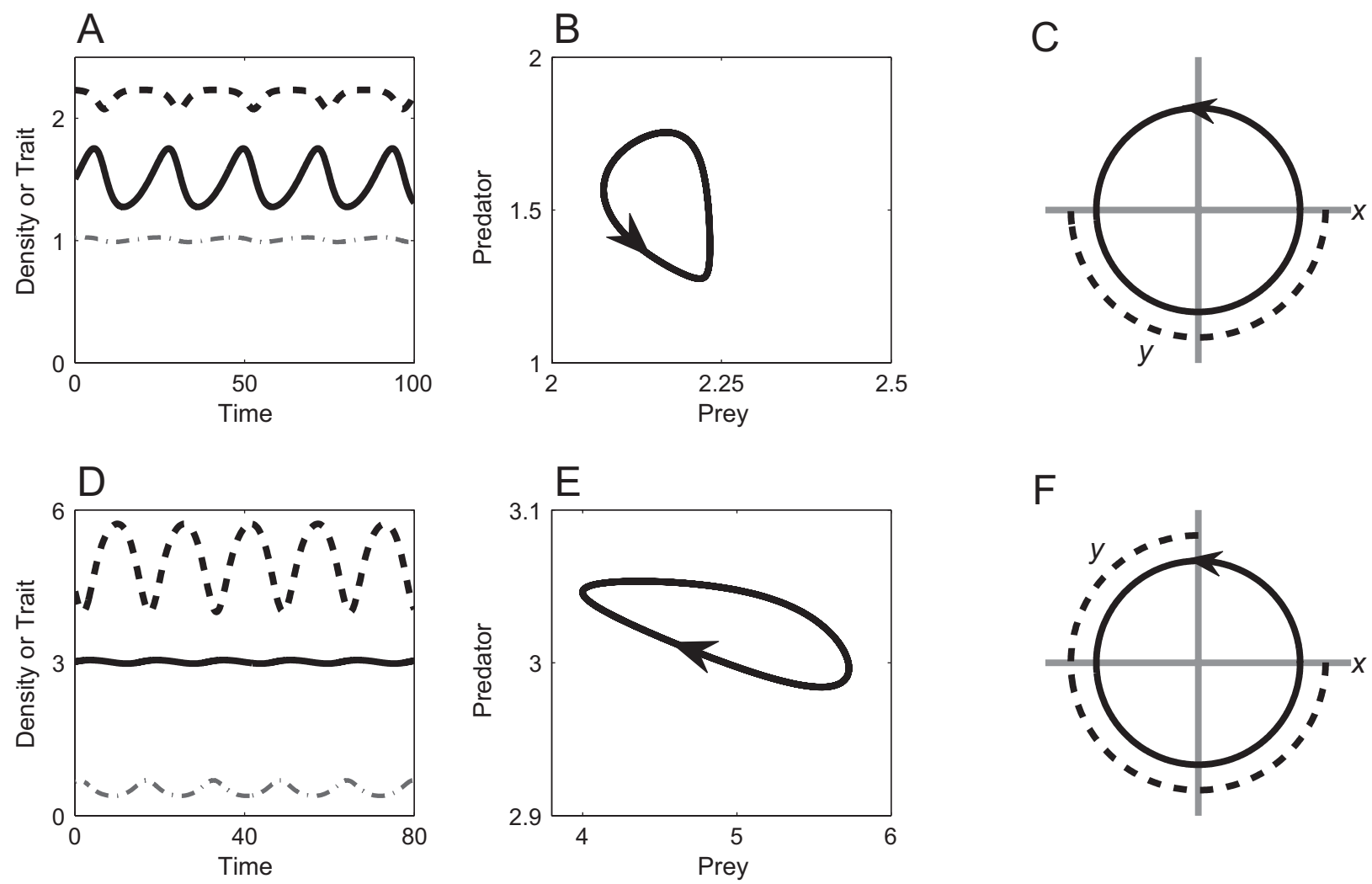

Figure 5: Predicted phase relations for predator-prey oscillations. Prey evolution can drive cycles with lags between one-quarter and onehalf of a period when the prey defense is very effective against predation $(A-C)$. In very rare cases, prey evolution can drive cycles with lags greater than a half period when increased prey density results in an increase in the selective pressure for defense $(D-F)$. $A, D$, Predator (solid black), prey (dashed black), and mean prey trait (dashed-dotted gray) time series. $B, E$, Population cycles in $A$ and $D$ projected onto the predator-prey plane. Arrows denote the flow of time. $C, F$, Predicted phase relations. The circle represents one period of the cycle, and the arrow denotes the flow of time; $x$ and $y$ denote when the prey and predator populations, respectively, reach their peak densities during the cycles in $A$ and $D$. The dashed arcs denote the range of phase lags observed in numerical simulations. See appendix E for models and parameter values.

in the ecological and evolutionary subsystems (e.g., stabilizing vs. disruptive selection) and the interactions between those subsystems. This work helps explain the different effects of increased prey genetic variation observed in experimental systems and identify connections between different theoretical approaches used to study eco-evolutionary dynamics.

My results help understand why increased prey genetic variation stabilized one rotifer-algal system (Steiner and Masse 2013) and destabilized another (Becks et al. 2010). Stabilization is predicted to occur when there is stabilizing selection $(\xi<0)$, and individual fitness increases with increased mean defense $\left(F_{\alpha}-G_{\alpha}<0\right)$. For the colonyforming defense in the Steiner and Masse (2013) study, this could occur, for example, if larger colonies decrease rotifer search and encounter rates and therefore decrease the consumption of algal cells in smaller colonies. Destabilization is predicted to occur when either (i) there is disruptive selec- tion $(\xi>0)$ or (ii) there is stabilizing selection $(\xi<0)$, and individual fitness decreases with increased mean defense $\left(F_{\alpha}-G_{\alpha}>0\right)$. Because the conditions for stabilizing selection are more restrictive - in agreement with the theoretical work in Becks et al. (2010)—my results predict that the destabilization in that study was driven by disruptive selection.

These predictions highlight how the interactions between the ecological and evolution subsystems (terms in the offdiagonal boxes of the Jacobian) can be important determinants of how increased genetic variation alters ecological dynamics. Under stabilizing selection, the sign and magnitude of the interaction term $F_{\alpha}-G_{\alpha}$ determines whether increased genetic variation is stabilizing $\left(F_{\alpha}-G_{\alpha}<0\right.$; fig. $4 A$, $4 B)$ or destabilizing $\left(F_{\alpha}-G_{\alpha}>0\right.$; fig. $\left.4 D, 4 E\right)$. In contrast, under disruptive selection destabilization occurs with increased genetic variation regardless of the sign of $F_{\alpha}-G_{\alpha}$. However, the sign of $F_{\alpha}-G_{\alpha}$ may have other effects on 
population-level dynamics when there is disruptive selection. In particular, the sign of $F_{\alpha}-G_{\alpha}$ influences the cost for prey defense: lower costs are expected when $F_{\alpha}-G_{\alpha}<$ 0 , and higher costs are expected when $F_{\alpha}-G_{\alpha}>0$. Previous studies have shown that costs for defense have other important effects on population-level dynamics. For systems under disruptive selection, lower costs for defense lead to cryptic cycles, and higher costs lead to antiphase cycles (Jones and Ellner 2007; Yoshida et al. 2007; Cortez and Ellner 2010). Kasada et al. (2014) observed that cyclic coexistence of multiple prey genotypes was possible when costs for defense were low; if costs for defense were too high, then evolution would drive the population to near fixation of the undefended genotype. Hence, while costs for defense do not affect whether disruptive selection destabilizes a system with increased genetic variation, they may have other important effects on population-level dynamics.

One important general conclusion of this study is that stability changes occur only when there is a sufficiently high amount of genetic variation. Specifically, system stability is determined by the ecological subsystem at low prey genetic variation, and that stability can be altered only if enough genetic variation is present in the prey population. To a first approximation, the critical magnitude of genetic variation is that which balances the (in)stabilities of the ecological and evolutionary subsystems. The Steiner and Masse (2013) and Becks et al. (2010) studies are not direct empirical tests of this prediction because the mean trait is not held (roughly) constant as prey genetic variation is increased. However, more direct tests can be done using similar methods. The key is to initiate different replicates of the same predator-prey system with different combinations of prey genotypes that lie along a trade-off curve (fig. 6). Prey genotypes with different levels of defense can be selected for by exposing prey to different constant levels of predation, as was done in Becks et al. (2010). This study then predicts that system stability will depend on the magnitude of prey genetic variation used to seed the system. For example, consider a predator-prey system with a clonal prey species where there is a concaveup trade-off between defense and growth rate (fig. 6A). The concavity of the trade-off curve implies that there is disruptive selection (i.e., the evolutionary subsystem is unstable; $\xi>0)$. Hence, if the system is stable in the absence of genetic variation, then the system is predicted to be stable when seeded with low amounts of genetic variation (fig. $6 B$, simulated using circle genotypes from fig. 6A) and to exhibit cycles when seeded with sufficiently high amounts of genetic variation (fig. $6 C$, simulated using square genotypes from fig. 6A).

Note that because of the generality of model (1), my results deal only with relative amounts of genetic variation (e.g., high vs. low). To identify the critical amount of genetic variation needed to (de)stabilize a particular system using the above-described experiments, one would need to create a model tailored to the system. For some cases, the amount of genetic variation needed for (de)stabilization may be larger than is biologically possible, implying that increased genetic variation may not have a qualitative effect on system stability. This is still an important conclusion, and it can be supported with theory by applying the methods and analysis described in the appendixes to the tailored model. For systems where (de)stabilization occurs for reasonable amounts
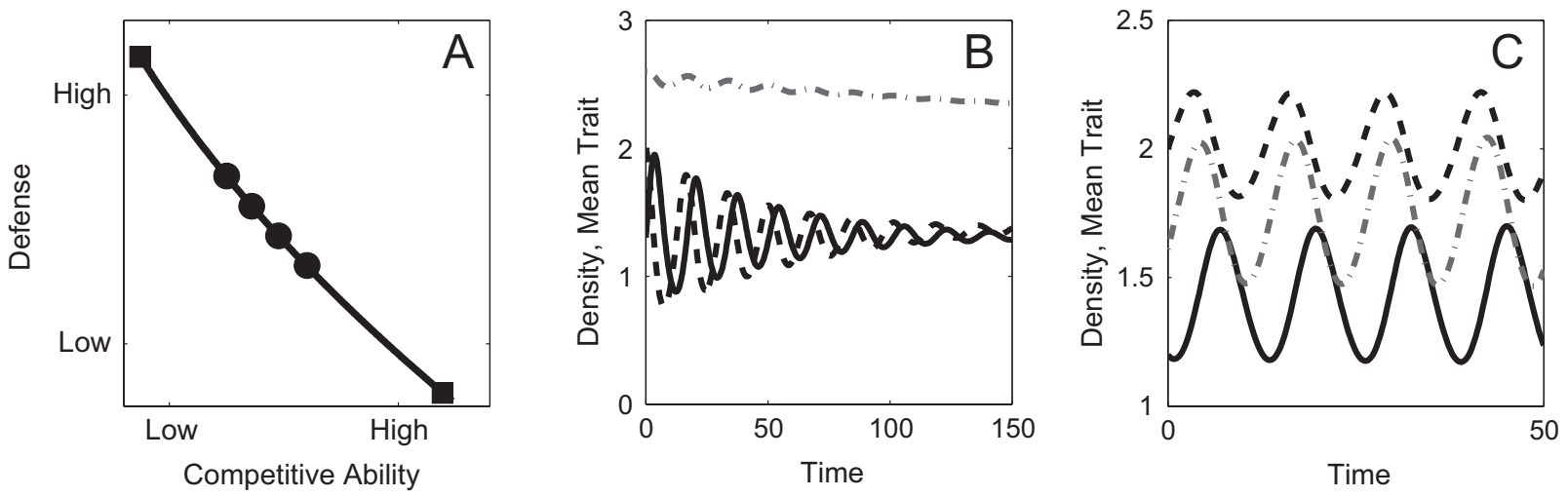

Figure 6: System stability is predicted to depend on the amount of additive prey genetic variation the prey population is seeded with. $A$, Prey genotypes (circles and squares) ordered along a curve defining the trade-off between growth rate and defense. $B, C$, Predator (solid black), total prey (dashed black), and mean prey trait (dashed-dotted gray) time series for a predator-prey system with two prey clonal types. The system is stable in the absence of prey genetic variation, and the concave-up trade-off curve implies that there is disruptive selection (i.e., an unstable evolutionary subsystem). In $B$, the system is stable when seeded with a low amount of genetic variation (genotypes denoted by circles in $A$ ). In $C$, oscillations occur when the system is seeded with a high amount of genetic variation (genotypes denoted by squares in $A$ ). See appendix $\mathrm{E}$ for model and parameter values. 
of genetic variation, the theory developed in this study can be used to identify what combinations of prey genotypes will be (de)stabilizing.

Previous studies have focused on how prey evolution can drive cycles where the phase lags are greater than onequarter of the cycle period (e.g., antiphase cycles; Yoshida et al. 2003; Jones and Ellner 2007; Cortez and Ellner 2010). Because such cycles are not predicted to arise in the absence of evolution (Bulmer 1975), they are predicted to be populationlevel signatures of prey evolution. My work adds support to this prediction. Specifically, my results show that if stability changes occur at low genetic variation, then the phase lags will be less than a quarter period (e.g., fig. 4C). In contrast, phase lags greater than a quarter period occur only when large magnitudes of genetic variation are needed to (de)stabilize a system. Altogether, this suggests that predator-prey phase lags greater than a quarter period are signatures of prey evolution and relatively high-standing prey genetic variation. Note that predator evolution (Cortez and Ellner 2010) and predatorprey coevolution (Mougi and Iwasa 2010, 2011; Cortez 2015) can also drive cycles with lags greater than a quarter period. Thus, one needs to rule out the possibility of predator evolution before making inferences about evolution and genetic variation in the prey.

While this study has focused on prey evolution, predatorprey coevolution can also alter population-level dynamics. In the limit where genetic variation is very low, coevolutionary cycles (Red Queen dynamics) can arise in systems that would be stable in the absence of evolution (Marrow et al. 1992, 1996; Gavrilets 1997; Khibnik and Kondrashov 1997; Doebeli and Dieckmann 2000; Dercole et al. 2003; Nuismer et al. 2005). Studies using quantitative genetic models with intermediate (Saloniemi 1993; Mougi and Iwasa 2010, 2011) or large (Cortez and Weitz 2014; Cortez 2015) amounts of genetic variation have also explored how coevolution can alter system stability and drive qualitatively different kinds of cycles. For example, coevolution can drive clockwise cycles where prey peaks follow predator peaks (Cortez and Weitz 2014; Cortez 2015); these cycles are the reverse of that predicted in the absence of evolution. Some of these studies (Saloniemi 1993; Gavrilets 1997; Doebeli and Dieckmann 2000; Mougi and Iwasa 2010, 2011) have explored how the relative amounts of genetic variation in the prey and predator populations affect the stabilities and dynamics of specific models; species coexistence, system stability, and cycle shape can all depend on the relative amounts of genetic variation. However, because of the specificity of the models, it is unclear what biological properties determine how predatorprey ecological and evolutionary dynamics are determined by the relative magnitudes of standing genetic variation in each population. The key to my approach is that I decompose the Jacobian $(J)$ into components representing the stabilities of the ecological and evolutionary subsystems and the interactions between those subsystems. This approach can be applied to any eco-evolutionary system. Hence, a similar approach may be helpful in identifying how the magnitudes of prey and predator genetic variation shape the ecological and evolutionary dynamics of eco-coevolutionary predator-prey systems. This approach may also be useful in extending results on how genetic variation influences eco-evolutionary dynamics in predator-prey systems with predator evolution (Abrams 1992, 2000), three-species systems with predator evolution (Schreiber et al. 2011; Patel and Schreiber 2015), coevolutionary competition systems (Agashe 2009; Mougi 2012), and three-species food-chain models with three coevolving species (Dercole et al. 2010).

\section{Connections with the Theory of Adaptive Dynamics}

One theoretical contribution of this work is that it begins to build connections between the stability conditions derived via the theory of adaptive dynamics (Marrow et al. 1996; Geritz et al. 1998) and approaches that assume larger amounts of additive genetic variation (Abrams and Matsuda 1997; Jones et al. 2009; Cortez and Ellner 2010). Importantly, while the quantitative genetic and adaptive dynamics frameworks make different biological assumptions, the equilibria of model (1) in the limit where $V$ is infinitesimally small $(V \ll 1)$ and models derived using the adaptive dynamics framework (Marrow et al. 1996; Geritz et al. 1998) are the same and have the same stabilities; see appendix A, section A3. Thus, analyzing model (1) allows one to identify connections between the stability results for the two bodies of theory. Here, I summarize the main points and refer the reader to appendix $\mathrm{D}$ for details.

To begin, I define three terms. First, as defined above, the evolutionary subsystem is stable if $\xi<0$ and is unstable if $\xi>0$. Second, an equilibrium trait value is an essential stable strategy (ESS) if it is an individual fitness maximum $\left(\left[F_{\alpha_{i} \alpha_{i}}-G_{\alpha_{i} \alpha_{i}}\right] / x<0\right)$; fitness minima $\left(\left[F_{\alpha_{i} \alpha_{i}}-G_{\alpha_{i} \alpha_{i}}\right] / x>0\right)$ are not ESSs. Third, an equilibrium trait value is convergent stable (CVS) if it is an attractor in the limit of very low genetic variation. Below, I focus on systems with stable ecological subsystems and CVS equilibria; for details, see appendix D. In the adaptive dynamics framework, evolutionary branching (i.e., speciation) occurs at CVS, non-ESS equilibria (Geritz et al. 1998).

Consider systems with unstable evolutionary subsystems $(\xi>0)$. For an equilibrium trait value that is a fitness minimum (not ESS), at low genetic variation the equilibrium point is stable (left side of fig. $3 A$ ), and the conditions for evolutionary branching are satisfied. With increased genetic variation (right side of fig. $3 A$ ), the system exhibits ecoevolutionary cycles. This means that destabilization caused by increased genetic variation could potentially inhibit speciation by causing cycles about a fitness minimum. Alterna- 
tively, if the equilibrium trait value is a fitness maximum (ESS), then increased genetic variation prevents the trait from converging to the fitness maximum and causes the trait to fluctuate about a fitness maximum. For systems with stable evolutionary $(\xi<0)$ subsystems, destabilization due to increased genetic variation can also cause the trait to cycle about a fitness minimum or maximum. The only difference is that the cycles can occur for all sufficiently large $V$ (fig. 4D) or for a range of intermediate values of $V$ (fig. $4 E$ ). In either case, the cycles may inhibit evolutionary branching at a fitness minimum (not ESS) or convergence of the mean trait to a fitness maximum (ESS). Examples of eco-evolutionary cycles about fitness minima and maxima have been discussed previously in Abrams et al. (1993).

These connections between adaptive dynamics-based and quantitative genetic-based theories help illustrate why both bodies of theory are needed to understand eco-evolutionary dynamics. For example, returning to the systems with unstable evolutionary subsystems discussed in the previous paragraph, quantitative genetic theory predicts for low genetic variation that evolution ceases once the system converges to the equilibrium point. However, it may be the case that evolutionary branching occurs, as predicted by the theory of adaptive dynamics.

Finally, adaptive dynamics and fast-evolution quantitative genetic approaches (e.g., Cortez and Ellner 2010) can be thought of as two extreme cases where standing genetic variation is very low or very high, respectively. Both are useful approximations for studying systems where the amount of standing genetic variation lies in between the extremes. My work helps identify under what conditions these two approaches yield complete and incomplete pictures of how eco-evolutionary feedbacks shape community-level dynamics. Specifically, when (de)stabilization occurs only for a single value of $V$ (figs. $3 A, 4 A, 4 D$ ), the two bodies of theory capture the full the set of possible dynamics. However, when (de)stabilization occurs for a finite range of values (fig. $4 B$, $4 E$ ), then the two bodies of theory yield an incomplete picture. Hence, while the infinitesimally small and infinitely large genetic variation limits are useful starting points for studying eco-evolutionary dynamics, they may yield an incomplete picture, and my approach provides a way to identify when those situations occur.

\section{Acknowledgments}

I thank three anonymous reviewers for helpful suggestions and comments on the manuscript.

\section{Literature Cited}

Abrams, P. A. 1992. Adaptive foraging by predators as a cause of predator-prey cycles. Evolutionary Ecology 6:56-72.
- 2000. The evolution of predator-prey interactions: theory and evidence. Annual Review of Ecology, Evolution, and Systematics 31:79-105.

2001. Modelling the adaptive dynamics of traits involved in inter- and intraspecific interactions: an assessment of three models. Ecology Letters 4:166-175.

. 2005. "Adaptive Dynamics" vs. "adaptive dynamics." Lournal of Evolutionary Biology 18:1162-1165.

Abrams, P. A., and H. Matsuda. 1997. Prey adaptation as a cause of predator-prey cycles. Evolution 51:1742-1750.

Abrams, P. A., H. Matsuda, and Y. Harada. 1993. Evolutionarily unstable fitness maxima and stable fitness minima of continuous traits. Evolutionary Ecology 7:465-487.

Agashe, D. 2009. The stabilizing effect of intraspecific genetic variation on population dynamics in novel and ancestral habitats. American Naturalist 174:255-267.

Becks, L., S. P. Ellner, L. E. Jones, and N. G. Hairston Jr. 2010. Reduction of adaptive genetic diversity radically alters eco-evolutionary community dynamics. Ecology Letters 13:989-997.

Bohannan, B. J. M., and R. E. Lenski. 1999. Effect of prey heterogeneity on the response of a model food chain to resource enrichment. American Naturalist 153:73-82.

Bolnick, D. I., P. Amarasekare, M. S. Araújo, R. Bürger, J. M. Levine, M. Novak, V. H. W. Rudolf, S. J. Schreiber, M. C. Urban, and D. A. Vasseur. 2011. Why intraspecific trait variation matters in community ecology. Trends in Ecology and Evolution 26:183-192.

Bulmer, M. G. 1975. Phase relations in the ten-year cycle. Lournal of Animal Ecology 44:609-621.

Cortez, M. H. 2015. Coevolution-driven predator-prey cycles: predicting the characteristics of eco-coevolutionary cycles using fastslow dynamical systems theory. Theoretical Ecology 8:369-382.

Cortez, M. H., and S. P. Ellner. 2010. Understanding rapid evolution in predator-prey interactions using the theory of fast-slow dynamical systems. American Naturalist 176:E109-E127.

Cortez, M. H., and J. S. Weitz. 2014. Coevolution can reverse predatorprey cycles. Proceedings of the National Academv of Sciences of the USA 111:7486-7491.

Dercole, F., R. Ferrière, A. Gragnani, and S. Rinaldi. 2010. Chaotic Red Queen coevolution in three-species food chains. Proceedings of the Royal Society B: Biological Sciences 277:2321-2330.

Dercole, F., J. Irisson, and S. Rinaldi. 2003. Bifurcation analysis of a prey-predator coevolution model. SIAM Journal of Applied Mathematics 63:1378-1391.

Doebeli, M. 1997. Genetic variation and the persistence of predatorprey interactions in the Nicholson-Bailey model. Journal of Theoretical Biology 188:109-120.

Doebeli, M., and U. Dieckmann. 2000. Evolutionary branching and sympatric speciation caused by different types of ecological interactions. American Naturalist 156:S77-S101.

Gavrilets, S. 1997. Coevolutionary chase in exploiter-victim systems with polygenic characters. Journal of Theoretical Biology 186:527534.

Geritz, S. A. H., É. Kisdi, G. Meszéna, and J. A. J. Metz. 1998. Evolutionarily singular strategies and the adaptive growth and branching of the evolutionary tree. Evolutionary Ecology 12:35-57.

Hughes, A. R., B. D. Inouye, M. T. J. Johnson, N. Underwood, and M. Vellend. 2008. Ecological consequences of genetic diversity. Ecology Letters 11:609-623.

Jones, L. E., L. Becks, S. P. Ellner, N. G. Hairston Jr., T. Yoshida, and G. F. Fussmann. 2009. Rapid contemporary evolution and clonal 
food web dynamics. Philosophical Transactions of the Roval Societv B: Biological Sciences 364:1579-1591.

Jones, L. E., and S. P. Ellner. 2007. Effects of rapid prey evolution on predator-prey cycles. Journal of Mathematical Biology 55:541-573.

Kasada, M., M. Yamamichi, and T. Yoshida. 2014. Form of an evolutionary tradeoff affects eco-evolutionary dynamics in a predatorprey system. Proceedings of the National Academv of Sciences of the USA 111:16035-16040.

Khibnik, A. I., and A. S. Kondrashov. 1997. Three mechanisms of Red Queen dynamics. Philosophical Transactions of the Roval Society B: Biological Sciences 264:1049-1056.

Lande, R. 1976. Natural selection and random genetic drift in phenotypic evolution. Evolution 30:314-334.

1982. A quantitative genetic theory of life history evolution. Ecology 63:607-615.

Marrow, P., U. Dieckmann, and R. Law. 1996. Evolutionary dynamics of predator-prey systems: an ecological perspective. Lournal of Mathematical Biology 34:556-578.

Marrow, P., R. Law, and C. Cannings. 1992. The coevoltuion of predator-prey interactions: Esss and Red Queen dynamics. Proceedings of the Roval Society of London B: Biological Sciences 250:133-141.

Mougi, A. 2012. Allelopathic adaptation can cause competitive coexistence. Theoretical Ecology 6:165-171.

Mougi, A., and Y. Iwasa. 2010. Evolution towards oscillation or stability in a predator prey system. Proceedings of the Roval Society B: Biological Sciences 277:3163-3171.

- 2011. Unique coevolutionary dynamics in a predator-prey system. Journal of Theoretical Biology 277:83-89.

Nuismer, S. L., M. Doebeli, and D. Browning. 2005. The coevolutionary dynamics of antagonistic interactions mediated by quantitative traits with evolving variances. Evolution 59:2073-2082.
Patel, S., and S. J. Schreiber. 2015. Evolutionary-driven shifts in communities with intraguild predation. American Naturalist 186:E98E110.

Pimm, S. L., and J. H. Lawton. 1977. Number of trophic levels in ecological communities. Nature 268:329-331.

Saloniemi, I. 1993. A coevolutionary predator-prey model with quantitative characteristics. American Naturalist 141:880-896.

Schreiber, S. J., R. Bürger, and D. I. Bolnick. 2011. The community effects of phenotypic and genetic variation within a predator population. Ecology 92:1582-1593.

Steiner, C. F., and J. Masse. 2013. The stabilizing effects of genetic diversity on predator-prey dynamics. F1000Research 2. doi:10.12688 /f1000research.2-43.v1.

Turelli, M., and N. H. Barton. 1994. Genetic and statistical analyses of strong selection on polygenic traits: what, me normal? Genetics 138:913-941.

Whitham, T. G., W. P. Young, G. D. Martinsen, C. A. Gehring, J. A. Schweitzer, S. M. Shuster, G. M. Wimp, et al. 2003. Community and ecosystem genetics: a consequence of the extended phenotype. Ecology 84:559-573.

Yoshida, T., S. P. Ellner, L. E. Jones, B. J. M. Bohannan, R. E. Lenski, and N. G. Hairston Jr. 2007. Cryptic population dynamics: rapid evolution masks trophic interactions. PLoS Biology 5:1-12.

Yoshida, T., L. E. Jones, S. P. Ellner, G. F. Fussmann, and N. G. Hairston Jr. 2003. Rapid evolution drives ecological dynamics in a predator-prey system. Nature 424:303-306.

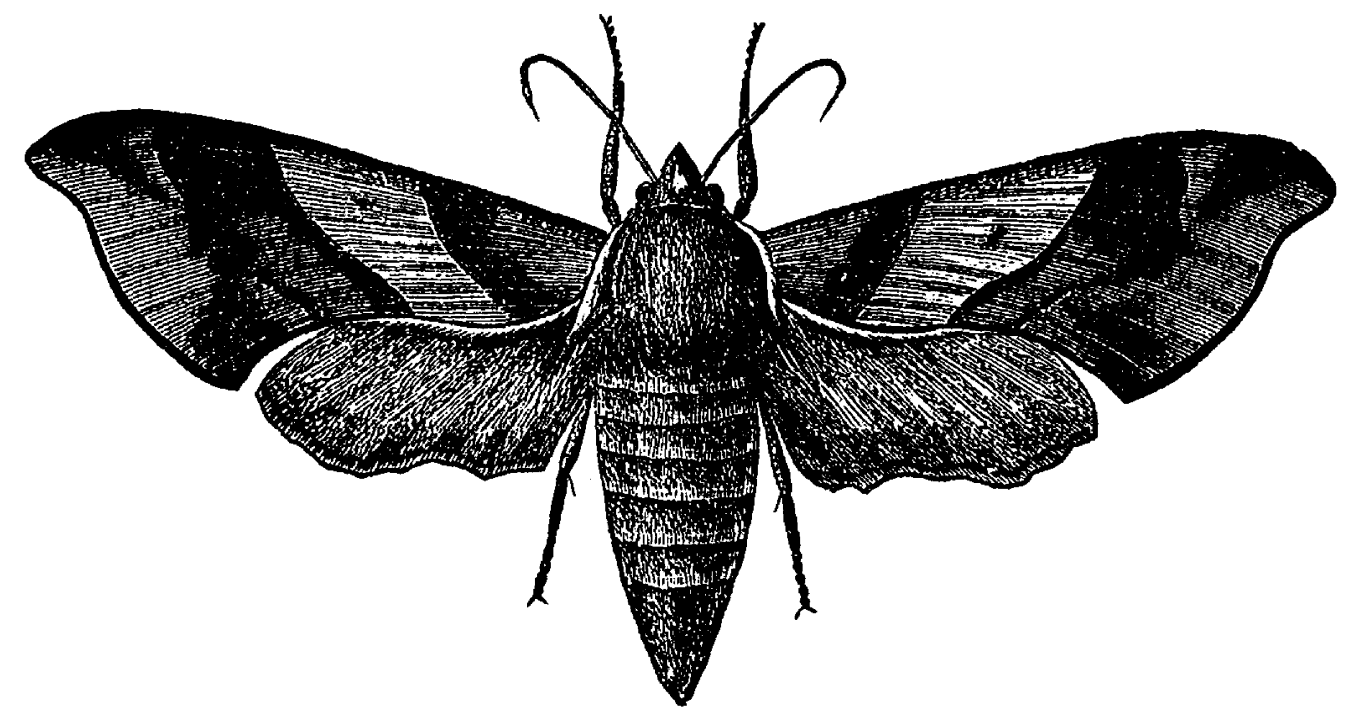

"The Vine dresser (Chœrocampa pampinatrix Smith and Abbott ... ), a single caterpillar of which will sometimes 'strip a small vine of its leaves in a few nights,' and sometimes nips off bunches of half-grown grapes." From the review "Economical Entomology in Missouri" (The American Naturalist, 1870, 4:610-615). 